\title{
Robotic Assembly System for Computer Integrated Construction
}

\author{
Thomas Bock, Division of Construction Automation, \\ University of Karlsruhe, Fasanengarten 3, D-76049 Karlsruhe, Germany
}

\begin{abstract}
Based on the experiences as a member of the working group "SMAS" within the programme entitled "Development of an Advanced Construction Technology by Applying the Electronic Technology" of the Japanese Ministry of Construction between 1985 and 88 the author proposed the R+D of a mobile robotic assembly system for computer integrated construction at the commission of the EU in 1991. The system is being developped between 1992 and 1996 and its intended marketing in 1998 should result in improuved quality, reduced construction time and cost within the german housing market. The various robotic and IT components are described in this paper.
\end{abstract}

\section{Introduction from SMAS to ROCCO}

The research project called "SMAS" (Solid Material Assembly System) for development of construction technologies supported by the Japanese Ministry of Construction programme, "Development of an Advanced Construction Technology by Applying the Electronic Technology", has been carried out through 1983 to 1988. (Please refer to the left picture at the end of this paper)

Components were positioned automatically by the robot one by one without arrangements of conventional bonding. Following the positioning of each components, steel bars were connected to those of adjacent components also by the the robot. The joint type of steel bar for vertical direction is mechanical, and that for lateral direction is overlapping. Concrete is grouted from the top of the wall which is erected one story high (about $3 \mathrm{~m}$ ). The newly developed endeffector by Hitachi Kenki was installed to the Fanuc robot, 6-articulation-type robot, which was developed for wide variety of applications in factory use. The characteristics of this system are mentioned below.

(1) The basic solid component that was stacked and composes structural walls, has been pre-cast concrete, $30 \times 30 \times 18 \mathrm{~cm}$ in size and weighed about $20 \mathrm{~kg}$.

(2) The solid components were stacked by the robot without filling mortal for bonding at their joints either vertical or lateral.

(3) For the reinforcement of the structural wall, the cross-shaped steel bars were fixed to each components and joined to each other by the robot when components are stacked. The robot grasped this steel fixtures and move the components to the designated position.

(4) The vertical joint was a screw-type mechanical joint and the lateral one was an over-lap type joint. 
(5) After the solid components were assembled, concrete grout were poured from the top of the wall and the components were filled with grout to comprise a wall of monolithic assembly.

(6) The foundation of the wall was cast-in-place concrete and floor may be made of wood, pre-cast concrete panels, cast-in-place concrete or others. A basic solid component was fitted with steel bars for the reinforcement to be assembled to a components-stacked wall.

For the verification of adequacy of the proposed over-lap type joint in lateral direction, wall specimens were made and the horizontal loading test was carried out. The result showed that the strength of the proposed joint is comparable to the ordinary lap-joint, when properly designed.

The SMAS featured the advantages of (1) leading to prefabrication while enhancing design flexibility since compact structural members are handled, (2) convenient application to rebuilding and retrofitting urban regions since it can work efficiently in areas of limited space, and (3) utilization as an integrated building production system since it can be linked with CAD/CAM systems. The above mentioned experiences resulted in the submission of an European $R+D$ project called "ROCCO" (RObotic assembly system for Computer integrated COnstruction) in 1991. In 1992 the ROCCO project started within the 3rd framework programme of ESPRIT and is being finalized in 1996.

\section{Robot Assemby System for Computer Intergrated Construction}

A predicted increased construction demand especially for low cost housing cannot be covered by today's constuction technologies and management methods. Furthermore there is a lack of skilled work forces $(50 \%$ of all foremen in Germany are over 50 years old). We can observe a drop of young talents in the construciton occupations and strong migration tendencies towards other branches. This is caused by very bad working conditions on the construction sites, which cannot withstand a comparison with industrial work places. A possibilty to meet the problem is the flexible automation in masonry construction, which brings rationalisation together with humanisation of the construction work.

The share of building construction in the total construction activities amounts to nearly $85 \%$ in Germany, where over the half falls the residential construction, which is again mainly masonry construction. Simultaneously the machine using rate in this field lies only between four and eight percent. Through construction robotics we can mobilize high rationalisation potentials by reducing high german labour- and overhead costs.

\subsection{Integral approach of the ROCCO project}

In any high wage country further rationalization can only be achieved by reduction of labour cost and construction time. Since the conventional mechanized methods for masonry have reached their system limits, they cannot contribute to further effectiveness enhancement. Therefore a certain innovative leap is required by a system approach that synchronizes existing construction technologies with new information and robotic technologies.

The system deals mainly with the construction oriented modification of existing technologies and with closing the gaps between them through intelligent interfaces and IT-based tools, in order to provide the necessary flexibility for one of a kind building production, robust design and user friendly programming. To achieve the 
requirements, the above mentioned basic strategies for automated construction are applied: the information integration, the transfer to the pre-fabrication (only where applicable) and the redesign of the used construction materials.

The main emphasis lies on the creation of automated system, which enables the complete and continuous automation and the integration of computer based construction systems, without restricting the freedom of the design of the architects. With that, one can build in a shorter time with fewer personnel more and better buildings.

\subsection{Functional profile of the ROCCO system}

Within the ROCCO project a mobile robot system will be developed for the assembly of masonry on-site.

Therefore a suitable robot under development as well as the integration in a computer based system for the working preparation and programming.

Figure shows shows the ROCCO total System.

The robot system with a reach of $5.50 \mathrm{~m}$ and a load capacity of $350 \mathrm{~kg}$ consist of a vehicle, the actual robot or manipulator and a gripping and assembly tool. In the framework of the working preparation, the necessary data for the pre-fabrication of the costumized blocks and for the robot programming are generated.

Based on a CAD representation of the building, first the walls are divided into the single blocks automatically by a software tool. The next step contains the planning of the construction site layout, i.e. calculation of the optimal working points of the mobile robot systems, the space for the pallets, the configuration of the blocks on the pallets and the sequences of the block's assembly. With the then available information the customized blocks for realizing individual wall dimensions can be produced, cutted and palletized on stationary plants. The last step of the working preparation is the generation of the robot programs.

\subsection{System requirements}

The system will be able to erect vertical walls with any dimension and openings on the construction site. Wall connections may occur under any angle and will be realized with the so called butt joint technique.

Basically all necessary process steps are considered:

- The placing of a possibly necessary levelling layer.

- The application of the necessary mortar

- The assembly of the building blocks

The concept of the total system provides the use of all market available and possibly slightly modified block systems and formats.

In the first step, only plane-parallel block systems will be used, which are assembled with thin bed mortar. After placing the first (levelling) layer, the adjusting effort is relative low. Respective blocks like sand-lime bricks, cellular concrete blocks or processed clay bricks with a plane parallel surface (so called "plane bricks") are best suitable for the automation.

All different wall thickness will be assembled: order neutral standard blocks as well as customized and cutted non-standard blocks will be processed.

The necessary standard blocks will be delivered on usual pallets. For the nonstandard blocks, special transport pallets are necessary, which differ slightly from 
the regular one. For the development of the prototype we assume that all blocks are placed vertically on the pallets.

Throughout the complete development, the boundary conditions on the construction site will be considered. Apart from climatic factors mainly the bearing capacity of the crane and of the floors and their accuracy will be taken into account.

\subsection{Construction site sequences}

With the above system, following working sequence on the construction site evolves:

The mobile robot system and the block pallets are placed on the floor by the crane in the rough initial positions determined by the working preparation. After referencing and measuring the position of the vehicle, the robot moves to the first working position. After positioning and supporting the vehicle, the actual position will be measured and compared to the planned position. The determined difference will be used as a compensating value inside the robot control, which compensates the off-line programmed commands.

The pallets, positioned with the construction site crane quite inaccurately, will be fine-positioned manually in the first step. But during the project also higher inaccuracies during gripping will be examined.

After gripping of the respective block from the pallet, the block will be placed by the manipulator in a rough position on the wall. The fine positioning will be executed by the gripping and assembling tool. So the gripping tool will compensate all the occurring small inaccuracies on the construction site.

\subsection{Robot system hardware}

The essential parts of the system are the vehicle, the manipulator and the gripping/assembling tool.

The manipulator. For an economical use, it is necessary to be able to assemble wall segments, which are big enough, on one working position in order to minimize the movements of the vehicle.

An examination showed, that $96 \%$ of all floors contains walls of $5.5 \mathrm{~m}$ height or lower. So that the robot system will be dimensioned that walls can be assembled until this height.

Bigger walls parts can be assembled as stair shaped segments. The picture below shows a wall of $3.5 \mathrm{~m}$ height with the segments able to build from one working position with two different block formats.

Additional to the resulting big working space of the manipulator it is necessary to move heavy blocks. The biggest on the market offered blocks have a weight up to $300 \mathrm{~kg}$. Additional the weight of the gripper has to be taken into account. So we come up to a handling weight of about $350 \mathrm{~kg}$. The combination of large reach and high load leads to hydraulic drives.

As kinematic concept, we choose a conventional, vertical, kinematic with two arms. Different examinations lead to that concept. With vertical walls and vertical palletized blocks, a concept with five axises is enough: three rotatory main axises and two rotatory wrist axises.

The vehicle. The vehicle moves the manipulator autonomously from one working point to another. The necessary navigation and security sensors are installed independently. The vehicle has a kinematic with four wheels, which are able to 
move on the rough floor surface also overcoming small obstacles with a height of 3 $\mathrm{cm}$. Additional the ground clearance is about $10 \mathrm{~cm}$.

Two diagonally mounted wheels, which are steered an driven, enable the vehicle to move in each direction with each orientation. Also turning on one point is possible. The vehicle has a load capacity of 2 tons, which are necessary to carry the manipulator and also some blocks during buffering. Four outriggers bring the necessary stability.

To navigate on the construction site an 2 dimensional external laser measurement system is installed. This is also used to measure the final working position to compensate the last minor positioning faults by the manipulator. Also levelling sensors are installed to measure the horizontal tilting error and delivers the value to an automatic levelling control actuating the outriggers.

The gripping and assembling tool. The tool consists of a positioning device and the actual gripper. Crucial for an exact positioning of the blocks in the wall is the positioning device. Through suitable sensors, passive compliant and active positioning elements the compensation off faults up to $\pm 40 \mathrm{~mm}$ is possible.

Additional a requirement is, that all different shapes of one block-system can be gripped without changing the tool to get low robot cycle times. This is realized by the use of a vacuum gripper, which gives the additional advantages to be able to integrate a damage control system.

The technology the thin mortar allows us to dip the block into the mortar, so that no extra mortar application device is necessary

\section{Integrated information management}

\subsection{Introduction}

The chosen approach bases on the idea of Computer Integrated Manufacturing (CIM), which is already successfully implemented in other industries and which shows there its efficiency. The idea is a continuous information flow from the architectural design to the automated execution of construction process on-site and in consideration of the construction elements.

This procedure, by us called Computer Integrated Construction (CIC), makes it possible to automatically process all once collected data without loosing the data consistency. This enables all participants to stay as flexible as necessary during short-term changes with as low error rates as possible.

The different development stages of the information flow between the parties participating in a construction process will be shown exemplary by means of the chosen application: masonry. The developed concept of CIC out of its leads into the components and tools described in the following chapters.

\subsection{Information flow}

Conventional information flow. Until the introduction of CAD-systems for designing buildings, all information necessary for masonry construction was included in the manually drawn architectural design plans. These plans where send to the executing construction company, who ordered with them the necessary building materials as non-costumized prefabricated standard products, adapted it according the plan sin a handicraft manner on construction site and assembled the walls manually based on the information of the architectural plans. 
This produce implied in each of its steps big sources for errors, which resulted in delays, subsequent work and bad quality of the construction.

Advanced flow information. In the last years, computer aided design gains more and more importance. The use of CAD in architectural offices is increasing rapidly and with this the availability of electronically processed data, which causes more exact and consistent plans. An additional trend can be observed in the building material industry. They are increasingly able to produce custamized non-standard blocks through computer aided working preparation and production, which can easily assembled on the site according to automatically generated assembly plans without the necessity of a manual re-shaping. Hereby the information flow is reversed by the building material industry. They get the information in prefabrication directly from the architect and pass the assembly plans and parts lists to the construction companies

$\because$ This procedure has some advantages compared to the conventional on:

- The omittance of the customizing and cutting of the blocks on the construction site.

- The better rationalization and humanization of the blocks' production and customization in the prefabrication through a higher grade automation.

- The optimized working sequences on the construction site through the assembly of pre-processed elements according to the assembly plans.

- The additional advantage of a better recyclability of the waste through cutting in the building material plants.

The "CIC" information flow:

To integrate the complete construction process in an IT-framework, as many as possible process steps should be based on electronical data processing. For the masonry we describe following a complete integrated information chain from the architect's design to the robotized execution of the tasks on-site, where all tasks are based on electronical data processing. This concept is IT backbone of the ROCCO project and represents the state of the art in European computer integrated construction systems.

The plans in the architectural offices are created with CAD-systems. On these base the production of standard and non-standard blocks in the pre-fabrication with the computer based production scheduling and numerically controlled production. This enables again the use of programmable assembly tools and systems on the construction site.

To use the architectural design data for production planning and programming in the prefabrication and on the construction site, it is necessary to process and to extend the data. The necessary software systems will be presented in the next chapter. The development of the software tools is realized within an interdisciplinary group on the base of already available knowledge.

\subsection{IT Systems components}

A basic difference between conventional robot applications, e.g. in mechanical engineering, and the robotized masonry is the number of repeatable robot motions. In conventional applications the same set of motions is repeated thousands of times, whereas every house has a unique design and every block has to be placed with an individually set of motions. However, at the same time we can reduce the structure of the motion process to some basic pattern like gripping, positioning, placing. This helps to reduce the complexity of the task.

13th ISARC 
A crucial importance has the programming of the robot. Normal robots can be teached or programmed with off-line programming-systems, where much more time is necessary to program the sequences than to execute it (but for the efforts are distributed to the number of cycles). This is not possible with a single piece production. Here an automatic program generation based on the available geometry data is compelling.

For this the start point on the pallet and the end point in the wall of each block must be known. Additional the position of the robot in relation to the floor must be known. This leads to another difference between industrial robots and on-site robots. The on-site robots are exposed to permanently changing environmental conditions. It is necessary to re-calibrate the robot at each working point. In addition unknown obstacles and events may occur, which make it necessary to adapt the robot easily to the environmental conditions.

During the whole working sequence, it is necessary, that the operator knows exactly, what statement is executed by the robot and how this can be manually modified, if environmental changes or material breaking occurs. The main difficulty lies in the re-entrance of the generated program after a manual modification, since the decision is necessary, at what point and with what parameters the program will re-start after unpredictable manual operations. To solve the problem, advance programming methods must be applied, which allow the simple and fast modification of the generated program.

We choose a distributed database approach to be able to run the different parts of the software system on different platforms (adapted to the respective requirements) and at different places, i.e. at the working preparation office, at the pre-fabrication plant and directly at the robot's working points on-site.

Off-line components. The used computer platform for the working preparation at the building material producer is a high level PC running the OS/2 operation system with the integrated OS/2 database manager. The relational database serves as the central storage and information distributor for following applications:

- Different CAD-format converters, which convert the architectural design information into a process-able format

- The graphical user interface, which enables the user to collect data from manually designed houses and to show the result $s$ of the following information processing systems.

- The wall partitioning software, which divides the architectural walls into the necessary blocks under the consideration of windows, doors, lintels, etc. The outputs are the dimensions and positions of each block in the respective walls. During the segmentation procedure, optimization criteria have to be considered under hard boundary conditions. The number of non-standard blocks should be minimized to keep the costs low and the dimensions should be well balanced to keep the waste during cutting low. Simultaneously official and technical prescriptions should be kept concerning the bearing capacities, the joints' positions, the walls' connection, etc.

- The sequence and task planning software, which is responsible for different calculation and optimization procedures. In the first step, the software has to determine the possible assembly sequences of the blocks, that is to generate an assembly precedence graph. In the second step, the optimal sequence has to be determined concerning the optimization criterion of minimizing the number of vehicle movements respectively of maximaizing the number of blocks built from 
one working position. This is mainly dependent on the reach of the manipulator. During the calculation of the optimal sequence, one can simultaneously determine the number and positions of the necessary working points. In the next step, this information is used to generate a collision -free path of the vehicle from the first working point to the last one along the sequence of working points.

- The palletizing software, which determines the position of the blocks on the pallets and the sequence and positions of the pallets on the construction site. Necessary information are the assemble sequences related to the perspective working points, the dimensions of the blocks and pallets, the position and dimension of the free storage space around the robot and the specific properties of the gripper, i.e. the gripping direction and for that the free reachable block surfaces. The main intention of the tool is to minimize the number of pallets under the condition of guaranteeing the necessary free reachable surfaces of the blocks for gripping. To achieve this goal advanced operation research algorithms as heuristics and genetic algorithms are applied.

After processing the incoming CAD-data with the above described tools, all necessary geometrical information as the positions and the dimensions of the blocks on the pallets and in the wall as well as the positions of the pallets and the robot system are available. Together with the assembly sequence, all data is onhand to be able to generate the control programs for the pre-fabrication production and cutting machines as well as for the on-site assembly robot system.

Pre-fabrication components. The operation in the pre-fabrication plant can be divided into two basic procedures. First the production of standard blocks in different formats. The production is not specific for a certain order. No customization is necessary. Therefore the only necessary information for the production scheduling is the number of blocks and their main format. Sop non specific software tool is necessary to process the data before using it for the production of standard blocks.

Another situation is in the production of the non-standard blocks as the second main procedure in the pre-fabrication. To get an efficient production of these blocks, it is necessary to have a continuos information flow from the above mentioned offline tools to the production machine controls. If one wants to assemble the tools automatically, not only the dimensions should be known for correct cutting but also the positions on the pallets for correct palletizing. To get an efficient production procedure, both, the cutting and the palletizing, should be automated. For both numerical controls with well-defined interfaces are necessary. In both cases, the geometrical information must be converted into motion information of the respective axises. Additional the sequence of cutting must be optimized to minimize the cuts and waste under the boundary conditions of the features of the used cutting equipment and of the succeeding palletizing station. So, three different software tools are necessary described below:

- The cutting sequence optimization, which minimizes the waste number of cuts considering different boundary conditions: The features of the used cutting equipment as the number of saw-blades or the thickness of the saw-blades, the features of the palletizing equipment as the ability to palletize randomly or the maximum number of the simultaneously available pallets. The software uses similar algorithms as the palletizing software in the one-line field. 
- The cutting program generation, which processes the geometrical information of the blocks into the motion information of the respective NC-controlled cutting axises.

- The palletizing program generation, which generates the motion programs for the palletizing devices. The generator has to consider not only the necessary final positions on the pallet. Depending on the design of the gripper, it is also necessary to consider the already palletized blocks in order to determinate the approach direction to avoid collisions.

The fully automated and computer-integrated pre-fabrication of the blocks represent an important prerequisite for a smooth assembly process on the construction-site. Construction site Components. After a conversion, the geometrical and process data are available in a format, which is suited to serve as the base for the robot program generation and the user interface for the robot control.

Here we chose an object oriented approach to implement a hierarchical implicit programming system to meet the following requirements with simultaneous ease of use, extendibility and programming power.:

Requirements: Integration of simulation packages, input and administration of world, sequence and program data, integration of path planning algorithms, providing of programming features, real time capability, interfaces to robot controls and measurement systems.

The system consists of an implicit layer, were the working sequence is described in an abstract, robot independent manner on three hierarchical levels: Mission, task, action. Each of the levels offers different commands to be used for the description of robot operation. With the representation of the different operations as icons in a precedence graph, it is possible to provide an easy to use programming feature without the necessity of the knowledge of a programming language. Also the automatic program generation is possible, since at the point only the assembly sequence information is necessary.

The second layer converts the implicit instructions into explicit elementary operations (EEOs), still robot language independent, but now provided with the explicit geometrical parameters for robotic motions. It is possible to integrate in this layer the automatic off-line planning, where the respective geometrical information can be used to parametrize each elementary move of the robot under the boundary conditions of a collision free path and consideration of the gripper features.

Through a succeeding interpreter layer, where the universal motion commands are transferred to the robot control dependent commands, it is possible to integrate different on-line control, sensor and simulation systems. Through the real-time process monitoring, it is possible to include sensor information in order to follow the execution and to update the world database even after switching from automatic to manual mode and vice-versa. One can integrate alternative manual operations either in the feed forward and nominal feedback line, if the assembly of difficult parts is necessary, or in the non-nominal feedback line, if the assembly of difficult parts is necessary, or in the non-nominal feedback line, if the sensor detect an irregularity or problem during execution.

This enables a flexible reaction to all unforeseeable situations on the construction site, which can be handled through manual re-programming of the automatic operation. 


\section{Summary}

The concept of the computer integrated construction allows the continuous automation of construction process, which was demonstrated with the masonry application. With the CAD-data of the designer as the base, it is possible to automate all working steps in the pre-fabrication as well as on the construction site without restricting the design freedom of the architect.

Through a careful definition of the necessary modules and interfaces we achieved a maximum flexibility. Each of the partial components is usable stand alone, e.g. the wall partitioning for the pre-fabrication of non-standard blocks (possibly with the cutting sequence optimization), the sequence and task planning for the optimizing of the manual masonry process, the implicit robot programming for advanced programming tasks in different fields etc.

Combined in an integrated system, the elements allow the realization of the IT-base to automate the masonry process with the known difficulties single piece production, changing and difficult environmental conditions. The system allows a consistent database handling throughout the complete construction process. This avoids errors during execution, increases the quality and is able to react flexibly during short term changes.

With the pilot realization in the masonry construction in the framework of the ROCCO project an example will be realized, which shows ways to a increased rationalization also in other fields of the overall construction process.

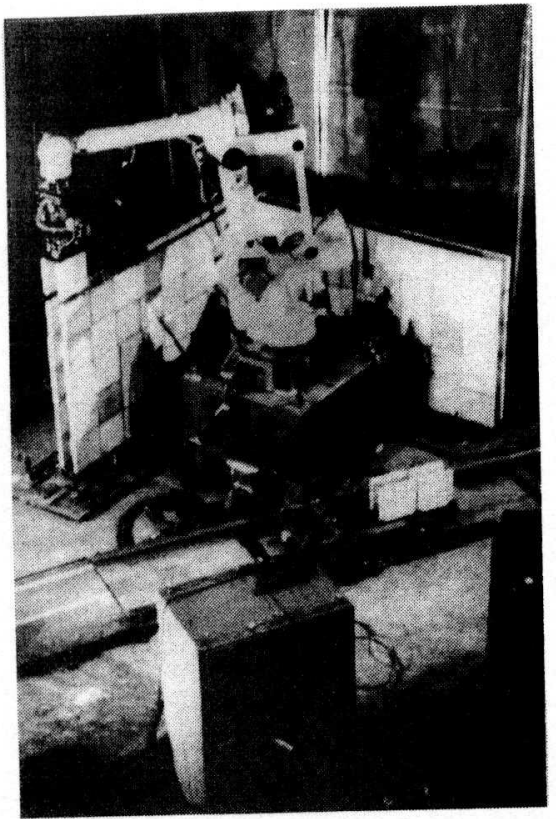

(1)

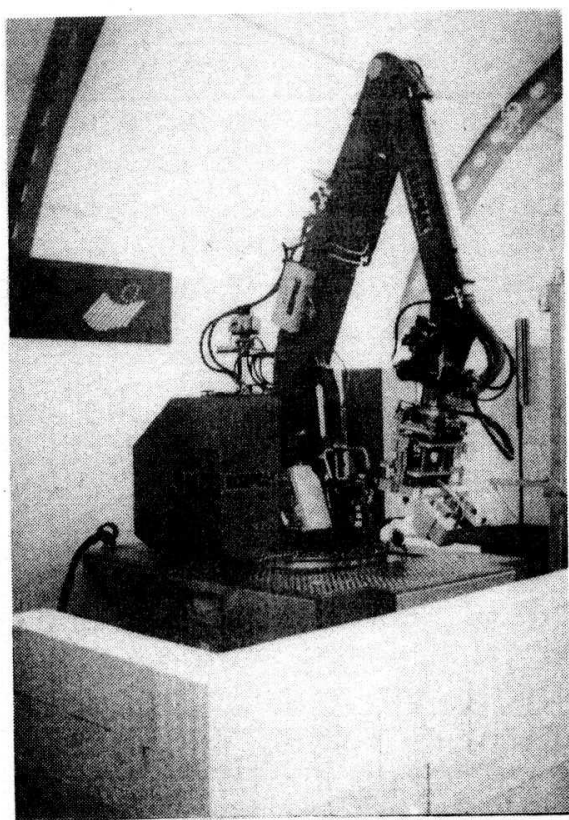

(2)

References:

(1) Working reports of SMAS project, Kokudo kaihatsu center , Tokio, 1985-88

(2) "Mauerwerksroboter und seine Integration in die Ausführungsplanung", Bautechnik, June 1995, Verlag Ernst und Sohn, Berlin 